\title{
Tratamiento de la anorexia y caquexia en el paciente terminal
}

\author{
F. J. Valcárcel Sancho
}

\section{Introducción}

Definimos como terminal, al paciente diagnosticado de cáncer en el que no existe posibilidad de un tratamiento oncológico potencialmente curativo pero en el que si es posible aplicar tratamientos paliativos. La expectativa de vida es de algunas semanas o meses. No nos vamos a referir a pacientes moribundos, con expectativa vital de pocos días, en los que raramente estará justificado un tratamiento para el síndrome anorexia-caquexia.

La importancia numérica y socio-económica del problema es grande. Actualmente, la incidencia de cáncer en España

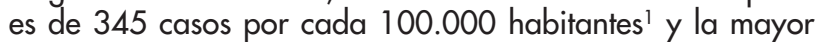
eficacia de los tratamientos han hecho aumentar la supervivencia de forma que aun en fases terminales, en muchos casos, esta se prolonga considerablemente. La frecuencia del síndrome anorexia-caquexia varía con cada tipo de tumor, pero hablando en términos generales, más de un 20 a $40 \%$ de los pacientes oncológicos presentan desnutrición en mayor o menor grado en el momento de su diagnóstico, y esta cifra puede superar el $80 \%$ en fases tardías de la enferme$\operatorname{dad}^{2-4}$. El paciente oncológico es absolutamente incapaz de mantener una adecuada ingesta calórica y proteica y va a desarrollar un círculo vicioso autoperpetuante y progresivo, que acaba produciendo una gran pérdida de masa corporal, agotamiento del tejido muscular, graso y de parénquimas. Con frecuencia, el síndrome anorexia-caquexia constituye uno de los principales problemas del paciente, cuando no el principal, y causa una gran ansiedad en el paciente y en su entorno familiar. Finalmente, se considera que un 20 a $25 \%$ de las muertes por cáncer derivan directamente de los efectos secundarios y complicaciones causadas por la desnutrición ${ }^{5}$.

Una encuesta nutricional recientemente elaborada en España (NUPAC-1) 6 que incluye entrevistas a 871 pacientes oncológicos de múltiples hospitales ha desvelado datos alar-

Servicio de Oncología Radioterápica Hospital Universitario Puerta de Hierro Madrid mantes: un $84 \%$ de los pacientes entrevistados requerían algún tipo de intervención nutricional; $68 \%$ de ellos tenían serios problemas para comer y $52 \%$ estaban moderada 0 intensamente malnutridos. El $67 \%$ consideraban subjetivamente la alimentación como muy importante (con la correspondiente repercusión en su calidad de vida). Finalmente se desprende de la encuesta que la mayoría no recibieron ningún tipo de soporte nutricional ni fueron valorados por un nutricionista.

\section{Causas de la malnutrición}

La causa del síndrome anorexia-caquexia es multifactorial. Hay una disminución del aporte de nutrientes por una menor ingesta calórica y fenómenos de malabsorción, anomalías metabólicas y condicionamientos psico-sociales. No entraremos en detalle a comentar la fisiopatología de la nutrición, desarrollada anteriormente en este mismo número, pero podemos resumir las causas de disminución de la ingesta en: 1.- las directamente relacionadas con el tumor, como obstrucciones del tubo digestivo, diarreas y vómitos, astenia, disgeusia, y dolor: 2.- los efectos secundarios de los tratamientos oncológicos que son importantes en el desarrollo de una ingesta disminuida: la cirugía oncológica, además de producir un aumento de requerimientos nutricionales causa desde alteraciones en la deglución a cuadros de diarrea o estreñimiento, pasando por cuadros de malabsorción mas o menos severa; la radioterapia, particularmente a nivel de cabeza y cuello conlleva importantes cuadros de mucositis con odinofagia, xerostomía, alteraciones dentarias y propensión a infecciones micóticas; en irradiaciones abdominales o pélvicas vemos cuadros de diarrea y malabsorción agudos o crónicos, y no son excepcionales los cuadros obstructivos o suboclusivos, que a su vez pueden requerir actuaciones quirúrgicas; la quimioterapia antineoplásica causa frecuentemente náuseas, vómitos y mucositis a nivel de todo el tubo digestivo con diarrea y malabsorción, y hepatotoxicidad con sus correspondientes repercusiones sobre el estado nutricional.

Hay alteraciones en el metabolismo de los carbohidratos con intolerancia a la glucosa y un consumo inefectivo de la misma lo que produce una disminución de sus niveles y disponibilidad. A nivel de lípidos, se produce un aumento de la lipolisis y disminución de la lipogénesis con la consiguiente disminución de las reservas de grasa. Se produce una dismi- 


\section{F. J. Valcárcel Sancho}

nución de la síntesis de proteínas y un aumento de su catabolismo con lo que disminuye la cantidad total de proteínas y atrofia de la piel y músculo estriado e hipoalbuminemia. Se implica además un aumento de determinadas citoquinas como el factor de necrosis tumoral alfa (TNF-_), interleuquinas 1 y 6, interferón gamma y otras como el factor inductor de proteolisis (PIE) y el factor movilizador de lípidos (LM) ${ }^{7-12}$.

No debemos olvidar los fenómenos psicosociales asocia$\operatorname{dos}^{13,14}$. El paciente terminal, además de tener que enfrentarse al diagnóstico de cáncer y a su posterior tratamiento experimenta cuadros de temor a la muerte, ansiedad y depresión que alteran los hábitos sociales y alimenticios del paciente. Igualmente, estos hábitos se ven alterados por el estrés existencial por la falta de cumplimiento de determinadas expectativas en la vida del paciente y la pérdida de su integridad personal, sociolaboral y familiar (sentimientos de ser una carga para la familia, empresa o sociedad) y la angustia ante una posiblemente temprana separación de sus seres queridos. Se desarrolla un cierto grado de aislamiento social al no poder participar en las actividades lúdicas y familiares en general asociadas a la comida. La cosa se agrava si es el propio paciente el que habitualmente compra y cocina sus propios alimentos. Su situación terminal le obliga a depender de su familia o del personal sanitario con una pérdida de control sobre su vida y decisiones. En los pacientes sometidos a nutrición enteral o parenteral hay además una pérdida de los placeres asociados a la comida cuando no otros problemas añadidos por la presencia de sondas nasoenterales, enterostomías, etc.

\section{Manejo clínico}

La malnutrición representa una causa importante de morbimortalidad en los pacientes oncológicos terminales y empeora notablemente su calidad de vida. Es frecuente que cause o que prolongue ingresos hospitalarios con el consiguiente deterioro del performance status y aumento del costo global del tratamiento oncológico.

Para la valoración nutricional del paciente oncológico terminal suele ser suficiente con una cuidadosa anamnesis dirigida, una exploración física meticulosa, y unas pocas determinaciones antropométricas como son el peso, la talla y el cálculo de índice de masa corporal (IMC = peso $(\mathrm{Kg}) / \mathrm{talla}^{2}$ $\left(\mathrm{m}^{2}\right)$. Las Escalas de Valoración Subjetiva Global la veces generadas por el propio paciente) se han demostrado de gran utilidad y alta fiabilidad. Su realización por los pacientes terminales a menudo cuesta un importante esfuerzo y pudieran ser menos apropiadas que en el enfermo oncológico con posibilidades de tratamiento curativo. Por último unas sencillas determinaciones bioquímicas, incluyendo albúmina, prealbúmina y en menor grado transferrina nos van a permitir una adecuada valoración basal y su modificación en el tiempo ${ }^{15-21}$. Para mayores detalles ver el capítulo dedicado a valoración nutricional en este mismo número.

Los objetivos que debemos perseguir en el tratamiento de estos pacientes serían como es obvio la corrección o prevención de déficits nutricionales, mejorar su calidad de vida y entorno familiar, evitar o acortar los ingresos hospitalarios y reducir las complicaciones derivadas de la desnutrición evitando un fallecimiento prematuro ${ }^{22}$. Otro objetivo, menos transcendente en el paciente terminal, sería mejorar la tole- rancia a los tratamientos oncológicos. Dada la condición de enfermo terminal en el que las medidas que apliquemos son paliativas es importante establecer una fluida comunicación con el mismo, dando prioridad a su opinión.

Podemos agrupar nuestras actuaciones en un adecuado tratamiento oncológico, una adecuada valoración e intervención nutricional, el aporte psicosocial posible, y una serie de medidas farmacológicas.

Se deberá emplear el tratamiento oncológico paliativo que sea posible y razonable en cada caso, con corrección de posibles obstrucciones, tratamiento del dolor, antidepresivos, etc.

Esto se simultaneará con una adecuada valoración e intervención nutricional, con estudio de los hábitos alimenticios y requerimientos nutricionales de nuestros pacientes, seguidas de una adecuación en la frecuencia y composición de la dieta, empleo de suplementos nutricionales, nutriciones enterales o parenterales.

No todo se reduce al empleo de determinadas dietas o suplementos nutricionales, el paciente oncológico demanda muy frecuentemente consejos en cuanto a su nutrición y en este sentido se ha comprobado como muy importante la consecución de un entorno adecuado a la hora de comer; se debe evitar someter al paciente a los olores producidos durante la preparación de las alimentos (frecuente causa de repugnancia y aversión a la comida en estos pacientes); es importante, siempre que se pueda, la realización de un determinado ejercicio físico (si se realiza antes de las comidas conseguiremos además evitar que el paciente esté sometido a los olores de la cocina, y podrá tomar algún aperitivo que estimula su apetito). Dentro del aporte social estaría que alguien pueda prepararle la comida y no sea el propio paciente el que la haga. En lo posible, deberán comer sentados en la mesa del comedor procurando un ambiente tranquilo y relajado, con tiempo suficiente para comer y evitando la presión familiar. En cuanto al contenido se procurará que los alimentos sean variados y con textura adaptada a cada situación. Se administrarán cantidades pequeñas repartidas en $5 \circ 6$ tomas al día, procurando realizar un mayor aporte calórico en los momentos (generalmente por la mañana) en que el paciente tolere mejor. Se puede añadir un aporte suplementario de proteínas y calorías sin un aumento importante del volumen de los nutrientes añadiendo en la preparación de la dieta: miel, nata, leche en polvo, crema de leche, helado, etc. Se deben evitar condimentos excesivos y temperaturas extremas. Se procurará una ingesta líquida abundante (alrededor de 2-3 litros al día) pudiendo ser al menos una parte de ella bebidas energéticas; para evitar la sensación de saciedad intentar que la ingesta de líquidos sea independiente de las comidas. En determinadas situaciones podremos recurrir al empleo de suplementos nutricionales que, al menos en fases iniciales no deberían sustituir a la ingesta de alimentos ${ }^{23-28}$

Cuando no es posible la alimentación convencional debidamente acoplada a las necesidades del pacientes podremos recurrir a algún tipo de nutrición enteral ${ }^{29-32}$ (ver el capítulo dedicado en este mismo número). Sus indicaciones estarían en aquellas alteraciones severas del tracto superior que impidieran una correcta alimentación natural, siempre que no haya obstrucción intestinal, síndrome de intestino corto o fístulas enterales. Las vías mas utilizadas consistirían en sondas nasogástricas o nasoyeyunales, seguidas de gastrostomías o yeyunostomías. Como complicaciones podemos encon- 
trar en primer lugar los problemas mecánicos derivados de la sonda como obstrucción de la misma, perforación a nivel del tubo digestivo y erosión a nivel nasal o de la faringe. Es posible la contaminación bacteriana de la dieta, sobre todo con preparados caseros triturados, y no es infrecuente ver cuadros de diarrea, estreñimiento, malabsorción o distensión abdominal así como alteraciones hidroelectrolíticas como hiperglucemia, hiponatremia, hipokaliemia e hipercalcemia. Podemos utilizar preparados caseros o recurrir a dietas comerciales de las que existe una gran variedad. En general, las dietas utilizadas en oncología tienen una proporción de carbohidratos menor y una mayor proporción de proteínas y de lípidos (que podrían por otra parte provocar saciedad precoz). Se pueden añadir según las necesidades de cada paciente determinados módulos nutricionales con hidrocarburos, lípidos, proteínas, aminoácidos, vitaminas, minerales - fibra, así como ácidos grasos $\omega-3$, fundamentalmente el ácido eicosapentaenoico (EPA) que además de mejorar el apetito y la capacidad funcional de los pacientes, estabiliza la pérdida de peso, revirtiendo la caquexia tumoral y modula la producción de citoquinas y la acción del sistema inmune. Es importante que el preparado elegido esté financiado por el SNS para no aumentar aun mas el quebranto económico de estos pacientes.

La nutrición parenteral raramente está indicada en el paciente oncológico terminal; su beneficio es mas que dudoso en los estudios randomizados y metaanálisis publicados y las complicaciones son frecuentes, tanto las derivadas del propio catéter (Neumotórax, hematomas, trombosis venosas de grandes vasos, sepsis, etc), como metabólicas (Alteraciones electrolíticas, de glucemia, insuficiencia cardiaca...). Así podríamos decir que sus indicaciones se limitarían muy concretamente a aquellos pacientes con alteraciones digestivas que tengan posibilidad de resolución aunque en un futuro no muy próximo y con una expectativa de vida razonable ${ }^{30-32}$.

Finalmente disponemos de un arsenal medicamentoso de gran utilidad.

La metoclopramida es un conocido antiemético que además acelera el vaciamiento gástrico, evitando las náuseas agudas y crónicas y la sensación de saciedad. Las dosis habituales de $10 \mathrm{mg}$ dos a cuatro veces al día pueden no ser suficientes en pacientes oncológicos que pudieran requerir dosis dos o tres veces superiores.

Los corticoides aumentan el apetito y la sensación de bienestar durante un corto período de tiempo en estos pacientes, pero no se ha demostrado ganancia ponderal, ni mejoría en el estado nutricional; además, sus conocidos efectos indeseables (edema, hiperglucemia, miopatía, toxicidad gastrointestinal, síndrome de Cushing, psicosis corticoidea, inmunodepresión, etc), limitan su uso como antianorexígenos, aunque a dosis de 4 a $8 \mathrm{mg} / \mathrm{d}$ de dexametasona o equivalente pudieran ser útiles en pacientes con expectativa vital corta ${ }^{33-36}$

El acetato de megestrol es un agente progestágeno que ha demostrado mejorar el apetito, la ingesta calórica y el estado nutricional, haciendo ganar peso no acompañado de retención hídrica y mejorando la calidad de vida en pacientes con cáncer. El efecto es visible a partir de una o dos semanas, por lo que es necesaria una expectativa vital de algunas semanas o meses. Es común que los enfermos que están perdiendo peso tarden alguna semana en dejar de perderlo, luego su peso se estabiliza y mas tarde aumentará. Muchos estudios randomizados ya clásicos ${ }^{37-41}$ demuestran repetidamente un significativo aumento del apetito, ingesta y peso con acetato de megestrol frente a placebo. Los efectos secundarios son poco habituales con las dosis estándar (Náuseas, vómitos o diarrea en un 5-7\%; por sus efectos hormonales impotencia, alteraciones menstruales o sangrado vaginal en 7 a $9 \%$ de los pacientes, edema maleolar leve en un $5 \%$ y ocasionalmente tromboflebitis profunda). Existe una cierta relación dosis-respuesta y se ha comprobado que con dosis de 160 mg/d ganan peso el 20 a 30\% de los pacientes, porcentaje que alcanza prácticamente el $100 \%$ con $1600 \mathrm{mg} / \mathrm{d}$. En estudios randomizados las dosis mas efectivas son de 600 a $800 \mathrm{mg} / \mathrm{d}$, sin que dosis superiores mejoren los resultados por lo que no se indican. Las dosis recomendadas actualmente irían de 160 a $800 \mathrm{mg} / \mathrm{d}$, según el efecto esperado y los resultados obtenidos ${ }^{42-45}$. Es el único fármaco autorizado en España para el tratamiento del síndrome anorexia-caquexia en pacientes oncológicos.

Se han empleado otros fármacos y sustancias en el tratamiento del síndrome anorexia-caquexia tumoral (Pentoxifilina, Ciproheptadina, Cannabis, Dronabinol, Hidralacina, Esteroides anabolizantes, $\mathrm{GH}$...), sin que actualmente haya evidencias concluyentes que avalen su utilización ${ }^{11,12}$.

Finalmente se están estudiando nuevas alternativas farmacológicas como serían la Melatonina, AINES (por su efecto inhibidor de la PGE2 y de la COX2), ATP, Bezafibrato, Inhibidores de la miostatina, Sensibilizadores de la Insulina, Inhibidores de citoquinas, etc, que no son de uso clínico actualmente ${ }^{11,12}$.

\section{Bibliografía}

1. Cancer Incidence, Mortality and Prevalence in European Union. European Network Cancer Registries (ENCR). http://wwwdep.iarc.fr/eucan/eucan.htm

2. Dewys WD, Begg C, Lavin PT, Band PR, Bennett JM, Bertino JR, Cohen MH, Douglass HO Jr, Engstrom PF, Ezdinli EZ, Horton J, Johnson GJ, Moertel CG, Oken MM, Perlia C, Rosenbaum C, Silverstein MN, Skeel RT, Sponzo RW, Tormey DC. Prognostic effect of weight loss prior to chemotherapy in cancer patients. Eastern Cooperative Oncology Group. Am J Med. 1980; 69:491-497.

3. Langstein HN, Norton JA. Mechanisms of cancer cachexia. Hematol Oncol Clin North Am 1991; 5:103-123.

4. Tisdale MJ. Cancer cachexia. Anticancer Drugs 1993; 4:115125.

5. Zeman FJ. Nutrition and cancer. In: Zeman FJ: Clinical Nutrition and Dietetics. 2nd ed. New York, Macmillan Pub. Co, 1991, pp 571-98.

6. A. Segura, C. Jara, J. Pardo, L. I. Zugazabeitia, J. Carulla, R. De Las Penas, S. Guevara, E. Garcia, E. Garcia, J. Casado. Epidemiological evaluation of nutritional status (NS) in patients (P) with locally-advanced or metastatic cancer (LAMC) ("NUPAC") Abstract No: 3120 Proc Am Soc Clin Oncol 22: page 776, 2003

7. Nelson KA, Walsh D, Sheehan A. The cancer anorexia-cachexia syndrome. J Clin Oncol, 1994; 12:213-225.

8. Tisdale MJ. Biology of Cachexia. J Natl Cancer Inst. 1997; 89:1763-1773.

9. Blaauw I, Deutz N, Meyenfeldt M. Metabolic changes in cancer cachexia ( first of two parts. Clin Nutr 1997; 16:169-176. 


\section{F. J. Valcárcel Sancho}

10. Blaauw I, Deutz N, Meyenfeldt M. Metabolic changes in cancer cachexia (second of two parts. Clin Nutr 1997; 16:223-228.

11. Invi A. Cancer Anorexia-Cachexia Syndrome. CA Cancer J Clin. 2002; 52:72-91.

12. Invi A. Cancer Anorexia-Cachexia Syndrome. Cancer Research. 1999; 59:4493-4501.

13. Jacobsen PB, Breitbart W. Psychosocial aspects of palliative care. Cancer Control Journal 1996; 3:214-222.

14. Cruzado JA, Labrador FJ. Intervención psicológica en pacientes de cáncer. Rev. Cancer 2000; 14:63-82.

15. Eldridge $B$, Rock $C L, M c C a l l u m ~ P D$. Nutrition and the patient with cancer. In: Coulston AM, Rock CL, Monsen ER, eds.: Nutrition in the Prevention and Treatment of Disease. San Diego, Calif: Academic Press, 2001, pp 397-412.

16. Dempsey DT, Mullen JL. Prognostic value of nutritional indices. J Parenter Enteral Nutr 1987; 11:109S-114S.

17. Dempsey DT, Mullen JL, Buzby GP. The link between nutritional status and clinical outcome: can nutritional intervention modify it? Am J Clin Nutr 1988; 47:352-356.

18. Sarhill N, Mahmoud FA, Christie R, Tahir A. Assessment of nutritional status and fluid deficits in advanced cancer. Am J Hosp Palliat Care. 2003; 20:465-473.

19. Ottery FD. Rethinking nutritional support of the cancer patient: the new field of nutritional oncology. Semin Oncol 1994; 21 : 770-778.

20. McMahon K, Decker G, Ottery FD. Integrating proactive nutritional assessment in clinical practices to prevent complications and cost. Semin Oncol 1998; 25: 20-27.

21. Bauer J, Capra S, Ferguson M. Use of the scored Patient-Generated Subjective Global Assessment (PG-SGA) as a nutrition assessment tool in patients with cancer. Eur J Clin Nutr 2002; 56:779-785.

22. Department of Health and Human Services. National Institutes of Health. National Cancer Institute. USA. Información en PDQ sobre cuidado médico para profesionales de la salud. Nutrición. http://www.cancernet.nci.nih.gov/clinpdq/supportive_span2/3 04467.html o http://www.medhelp.org/lib/cancernet/304467.htm

23. National Cancer Institute. Eating Hints for Cancer Patients: Before, During \& After Treatment. Bethesda, Md: National Cancer Institute, 1998. Publication No. 98-2079.

24. Stratton RJ. Summary of a systematic review on oral nutritional supplement use in the community. Proc Nutr Soc 2000; 59:469-476.

25. Tait NS. Anorexia-cachexia syndrome. In: Yarbo $\mathrm{CH}$, Frogge MH, Goodman M, eds. Cancer Symptom Management. 2nd ed. Sudbury, Mass: Jones and Bartlett Publishers, 1999, pp 183-197.

26. Ottery FD:.Supportive nutrition to prevent cachexia and improve quality of life. Semin Oncol 1995; 22(2 Suppl 3):98-111.

27. Farmer G: Pass the Calories, Please! A Cookbook and ProblemSolving Guide for People Who Need To Eat More. Chicago, III: The American Dietetic Association, 1994.

28. Recomendaciones nutricionales generales para el paciente oncológico. http://www.senba.es/recursos/cancer/presentac.htm

29. Piazza-Barnett $R$, Matarese LE. Enteral nutrition in adult medi$\mathrm{cal} /$ surgical oncology. In: McCallum PD, Polisena CG, eds. The Clinical Guide to Oncology Nutrition. Chicago, III: The American Dietetic Association, 2000, pp 106-118.

30. DeChicco RS, Steiger E. Parenteral nutrition in medical/surgical oncology. In: McCallum PD, Polisena CG, eds. The Clinical Gui- de to Oncology Nutrition. Chicago, Ill: The American Dietetic Association, 2000, pp 119-125.

31. Bozzetti F, Braga M, Gianotti L, Gavazzi C, Mariani L. Postoperative enteral versus parenteral nutrition in malnourished patients with gastrointestinal cancer: a randomised multicentre trial. Lancet 2001; 358:1487-1492.

32. Shils $M E$, Olson JA, Shike $M$, et al., eds.: Modern Nutrition in Health and Disease. 9th ed. Baltimore, Md: Williams \& Wilkins, 1999.

33. Moertel CG, Schutt AJ, Reitemeier RJ, Hahn RG. Corticosteroid therapy of preterminal gastrointestinal cancer. Cancer. 1974; 33:1607-1609.

34. Willox JC, Corr J, Shaw J, Richardson M, Calman KC, Drennan $M$. Prednisolone as an appetite stimulant in patients with cancer. Br Med J. 1984; 288:27.

35. Bruera E, Roca E, Cedaro L, Carraro S, Chacon R. Action of oral methylprednisolone in terminal cancer patients: a prospective randomized double-blind study. Cancer Treat Rep. 1985; 69:751-754.

36. Popiela T, Lucchi R, Giongo F. Methylprednisolone as palliative therapy for female terminal cancer patients. The Methylprednisolone Female Preterminal Cancer Study Group. Eur J Cancer Clin Oncol. 1989; 25:1823-1829.

37. Loprinzi CL, Ellison NM, Schaid DJ, Krook JE, Athmann LM, Dose AM, Mailliard JA, Johnson PS, Ebbert LP, Geeraerts LH. Controlled trial of megestrol acetate for the treatment of cancer anorexia and cachexia. J Natl Cancer Inst. 1990; 82:1127-1132.

38. Tchekmedyian NS, Hickman M, Siau J, Greco FA, Keller J, Browder H, Aisner J. Megestrol acetate in cancer anorexia and weight loss. Cancer 1992; 69:1268-1274.

39. Bruera E, Macmillan K, Kuehn N, Hanson J, MacDonald RN. A controlled trial of megestrol acetate on appetite, caloric intake, nutritional status, and other symptoms in patients with advanced cancer. Cancer 1990; 66:1279-1282.

40. Feliu J, Gonzalez-Baron M, Berrocal A, Artal A, Ordonez A, Garrido P, Zamora P, Garcia de Paredes ML, Montero JM. Usefulness of megestrol acetate in cancer cachexia and anorexia. A placebo-controlled study. Am J Clin Oncol. 1992; 15:436-440.

41. MA Segui, C Vadell, JM Gimenez-Arnau, S Morales, LI Cirera, I Bestit, E Batiste, R Blanco, L Jolis, M Boleda, I Anton. Doubleblind randomized trial for the treatment of cancer related cachexia: comparison of placebo vs two different doses of megestrol acetate. Abstract No: 1708 ASCO Annual Meeting 1996

42. Loprinzi CL, Bernath AM, Schaid DJ, Malliard JA, Athmann LM, Michalak JC, Tschetter LK, Hatfield AK, Morton RF. Phase III evaluation of 4 doses of megestrol acetate as therapy for patients with cancer anorexia and/or cachexia. Oncology. 1994; 51(Suppl 1):2-7.

43. Loprinzi CL, Michalak JC, Schaid DJ, Mailliard JA, Athmann LM, Goldberg RM, Tschetter LK, Hatfield AK, Morton RF. Phase III evaluation of four doses of megestrol acetate as therapy for patients with cancer anorexia and/or cachexia. J Clin Oncol. 1993; 11:762-767.

44. Cruz JM, Muss HB, Brockschmidt JK, Evans GW. Weight changes in women with metastatic breast cancer treated with megestrol acetate: a comparison of standard versus high-dose therapy. Semin Oncol. 1990; 17(6 Suppl 9):63-67.

45. Muss HB, Case LD, Capizzi RL, Cooper MR, Cruz J, Jackson D, Richards F 2nd, Powell BL, Spurr CL, White D, et al. High- versus standard-dose megestrol acetate in women with advanced breast cancer: a phase III trial of the Piedmont Oncology Association. J Clin Oncol. 1990: 8:1797-1805. 